\title{
Real-time language comprehension research using the Apple-Psych system
}

\author{
MARK BEEMAN and MORTON A. GERNSBACHER \\ University of Oregon, Eugene, Oregon
}

\begin{abstract}
We describe the use of the Apple-Psych system to conduct language comprehension experiments. Using this system, we have implemented several tasks commonly used to assess the cognitive processes involved in language comprehension. These include segment-by-segment reading tasks in which the stimuli are presented visually, and divided-attention and crossmodal tasks in which the stimuli are presented both visually and auditorily. Currently we are using the system to control collection of event-related potentials while subjects comprehend auditory discourse.
\end{abstract}

This paper describes a few of the many laboratory tasks that can be implemented on the Apple-Psych system to study language comprehension. An overview of the primary components of the Apple-Psych system is available in Barnes and Burke (1988) and Osgood (1988). A more complete description is provided in Osgood's (1984a) Hardware Implementation and Utility Manual and his (1984b) Software Program Development Guide. Both are available from Gil Osgood, the author of the ApplePsych system. ${ }^{1}$

We have used the Apple-Psych system with the following hardware: an Apple IIe, the required Mountain Computer clock card, and the optional parallel I/O card for collecting subjects' responses. We have modified this setup so that we can test 4 subjects simultaneously. The modification involved daisy-chaining the video output to five monitors, one for the experimenter and one for each of 4 subjects. Through the 8 -bit parallel $1 / 0$ card, we simultaneously collect binary responses from each of the 4 subjects. We have arranged our laboratory so that each subject sits at an individual booth, and each booth is equipped with its own monitor, audio headphones, and a two-key response board. This arrangement is illustrated in Figure 1.

We describe here how we have used this system to conduct experiments that employ various language comprehension tasks common to psycholinguistic research. All of the tasks involve presenting language stimuli: words, phrases, sentences, or paragraphs. In some of the tasks, the stimuli are presented visually on the subjects' video monitors. In other tasks, the primary stimuli are presented auditorily from a reel-to-reel tape player to the subjects' headphones, and the secondary stimuli are presented visually on the subjects' monitors. In some of the tasks, we measure how long it takes subjects to comprehend a unit of text, for example, a word, phrase, or sentence. In other tasks, we measure how long it takes subjects to

Preparation of this paper was made possible by National Science Foundation Grant BNS 85-10096. Address correspondence to Mark Beeman, Department of Psychology, University of Oregon, Eugene, OR 97403. respond to a secondary stimulus, for example, to respond when a tone occurs or to verify whether a particular word has occurred in the text. Thus, in all of the tasks, the primary dependent variable is subjects' response times, although we also measure accuracy of response when the subjects are making binary responses (e.g., deciding whether a particular word occurred).

\section{All-Visual Tasks}

The simplest tasks involve presenting text from a stimulus file onto the subjects' monitors. These are all-visual tasks. There are many ways that the text can be presented on the video monitors. Typically, the text is presented one unit at a time. These units may be words, phrases, or sentences. When presenting text one word at a time, which is becoming one of the most popular methodologies (Just, Carpenter, \& Woolley, 1982), one can vary where on the video monitor the words appear. In one variant of this task, each consecutive word appears in the center of the screen, replacing the previous word (e.g., Aaronson \& Scarborough, 1976; Juola, Ward, \& McNamara, 1982). In another variant, each word appears on the screen at its successive naturally occurring position. In addition, the successively appearing words can accumulate on the screen, or as each word appears, the previous word can disappear (a moving window display). An algorithm for creating a moving window display using the Apple-Psych system is presented in Listing $1 .^{2}$

In Listing 1 , the presentation duration of each word is prespecified by the experimenter (i.e., the values of Wordon and Wordoff). One method for assigning presentation durations is to make them a function of the word's length (see Just et al., 1982). We have used the following algorithm: a constant of $250 \mathrm{msec}$ per word plus $16.667 \mathrm{msec}$ per character.

In contrast to having the presentation duration under the experimenter's control, it can be under the subject's control; that is, each stimulus word can remain visible until the subject presses a response key. In this way, one can measure how long the subject needs to read each successive word of text. ${ }^{3}$ This can be implemented in the 


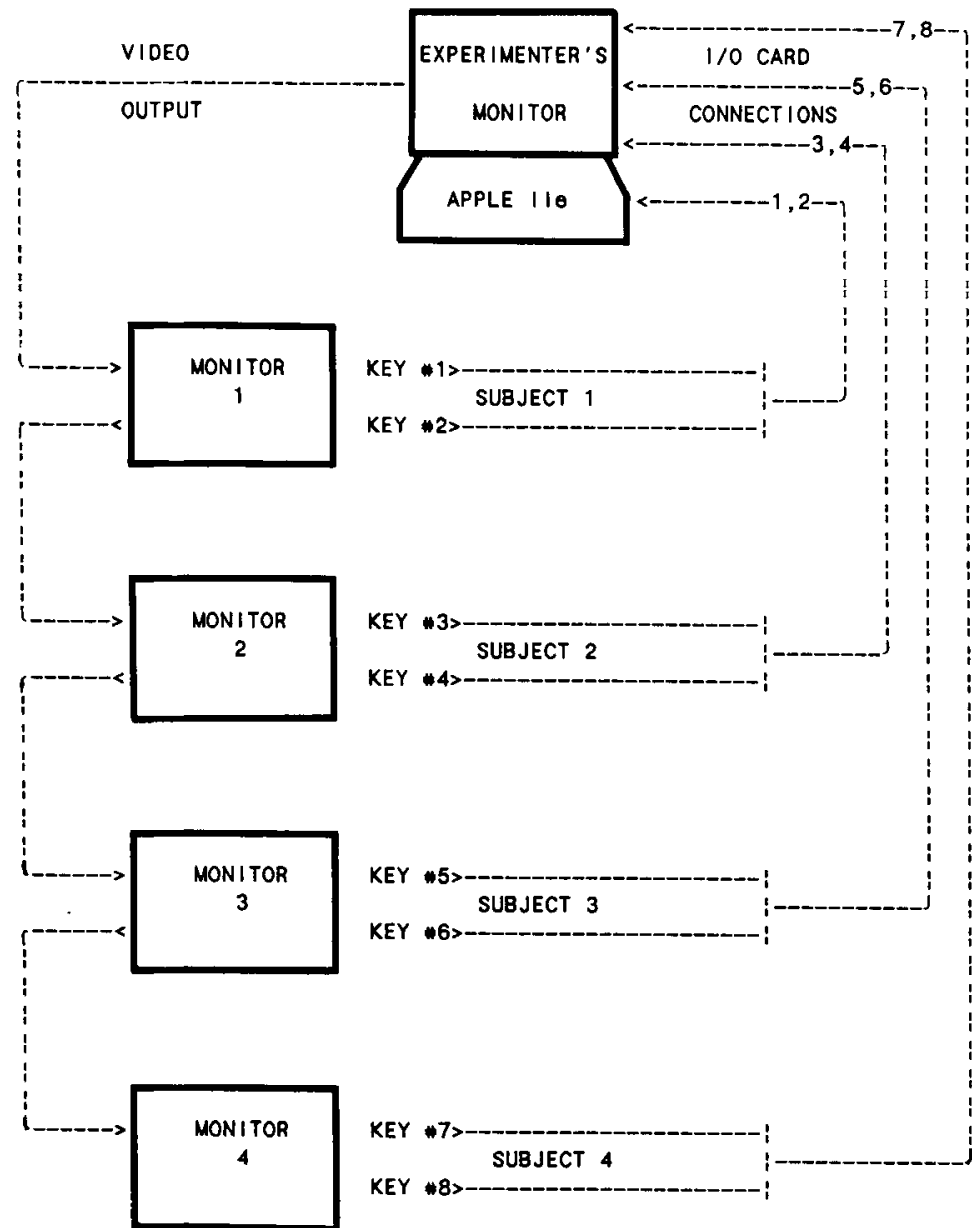

Figure 1. Schematic representation of the interfacing necessary to test 4 subjects simultaneously using Apple-Psych.

LISTING 1

Algorithm for Creating a Moving Window Display

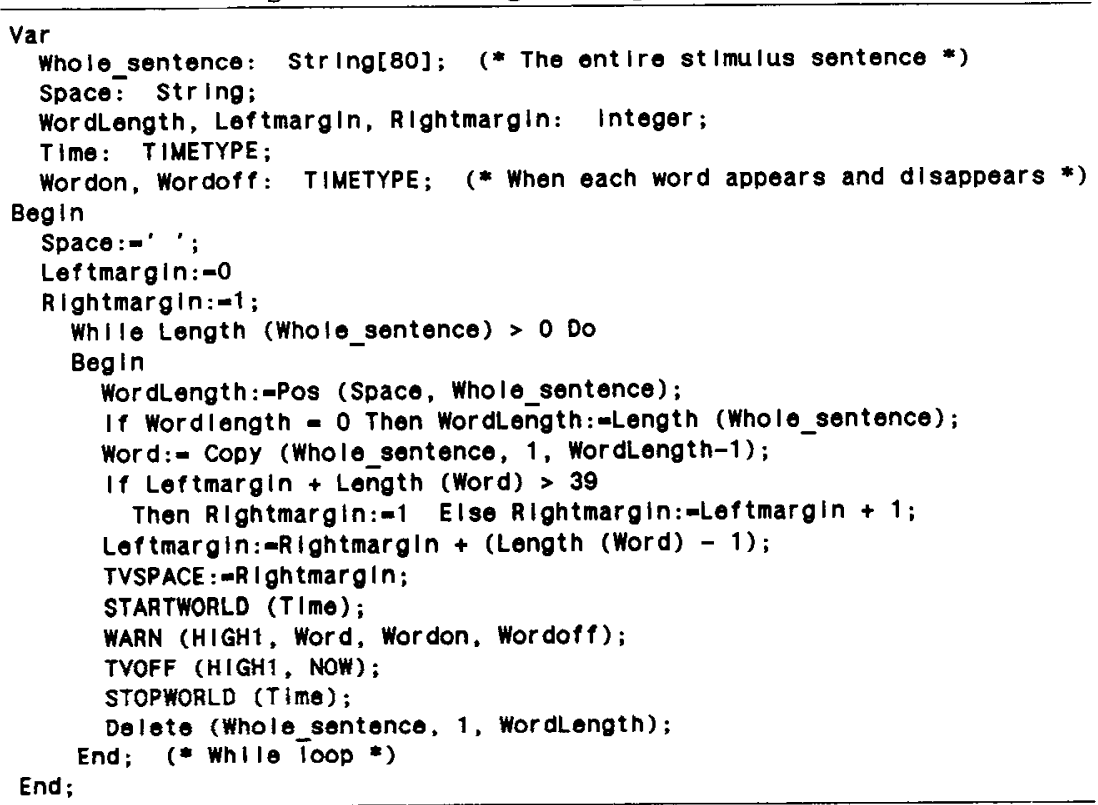


above example by using a SHOW followed by a GETRESP instead of a WARN. The above example can also be changed from a moving window display to a centered word-by-word presentation by simply changing the TVSPACE $:=$ Rightmargin to TVSPACE: $=0$ (thereby eliminating the need to compute left margin and right margin).

Reading time alone can be used to measure comprehension, or successive measures of comprehension, such as recall or recognition, can be taken (see Chang, 1983, and Levelt, 1978, for reviews). Other measures of comprehension can be gathered by requiring subjects to divide their attention between two tasks.

\section{Divided-Attention Tasks}

Divided-attention, or dual-task, methodologies provide an additional index of comprehension processing or difficulty by adding another task and measuring response times for it. Subjects respond when a prespecified target (or probe) occurs. These probes can be internal to the language stimuli, such as a specific word or letter, or external, such as a flash of light or a symbol. It is assumed that the response times to these secondary probes will increase whenever the difficulty of the primary stimulus, the text, increases.

In a related type of task, the probe items tap the subject's ongoing mental representation of the text that he/she is comprehending. For example, while subjects are reading a text, they can simultaneously perform a lexical decision task. In a lexical decision task, a string of letters appears on the screen. The subjects' task is to decide rapidly whether that string of letters forms a word. This task has been used primarily to measure what other concepts may be "activated" or strongly accessible in the comprehender's mental representations while he/she is comprehending the text. For example, while reading a sentence about airports, related concepts such as ticket counters and planes may also be activated in the comprehenders' mental representations. The more activated the concept, the faster the subjects' reaction times to make the lexical decisions should be.

Another method is to present a word during the ongoing text and ask subjects to verify whether that word has occurred in the text (see Chang, 1980). Again, the justification is that the more activated the concept, the faster the subjects' reaction time. Both the lexical decision and verification tasks can be implemented with the Apple-Psych system by first creating a program that presents the text word by word (as in the example code presented above). Then, the probe word (the word to which subjects make lexical decisions or verification judgments) can appear at any point in the sentence. One simply has to mark that word in the stimulus file, which can be done by specifying a particular first character, for instance, an asterisk. To signal the subjects which word of the sentence it is to which they are supposed to make lexical decisions or verification judgments, one can either present the word in a different location on the screen (e.g., at the top of the screen as opposed to the center; as in Chang, 1980) or flank the probe word with asterisks (as in Kintsch \& Mross, 1985). Both of these implementations can be made by adding the program code illustrated in Listing 2 to the program code illustrated in Listing 1.

\section{Crossmodal Tasks}

Crossmodal dual tasks can also be used to study the mental processes underlying language comprehension. One type of crossmodal dual task involves presenting text visually and having subjects perform an auditory secondary task. One such task involves subjects' reading the text from the video monitors and simultaneously monitoring for a tone. The text is presented via a modified moving window. This is called a modified moving window because, unlike the moving window display described above, the window is irrespective of word boundaries. Rather, a window of a prespecified size is chosen, for instance, one of 12 characters, and this window remains

LISTING 2

Code for Signaling Subjects Which Word Requires Response

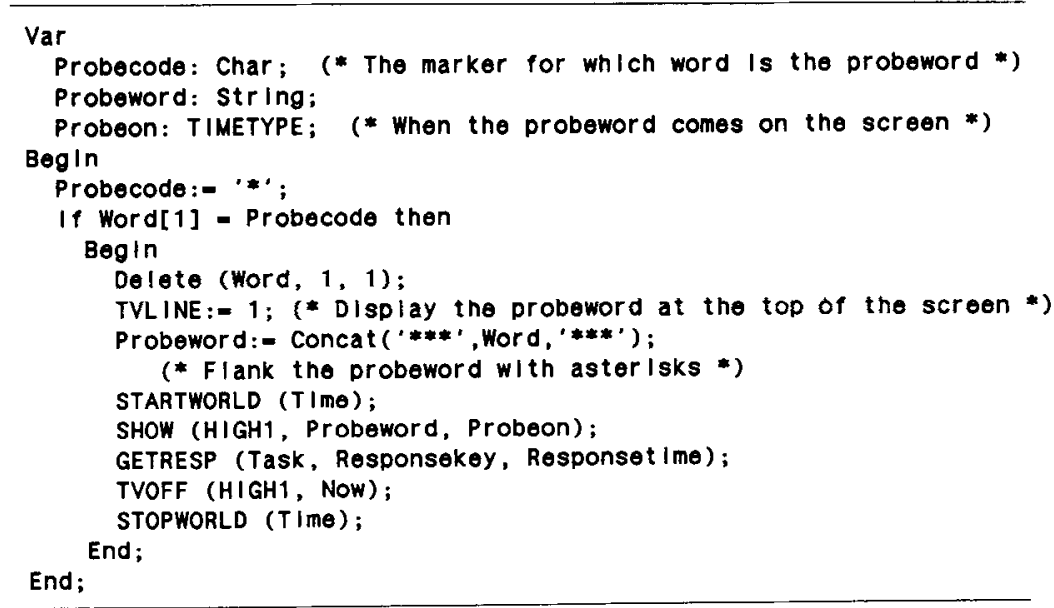


constant. So, at each point in the text presentation, 12 characters (including spaces between words) are visible. The appearance of the moving window is created by simultaneously adding 1 character to the right end of the display and removing 1 character from the left end. In addition to varying the width of the window, one can also vary the speed of window movement. This variation in effect produces different reading rates. Sometimes when this modified moving window display is used, we allow subjects to choose the speed (or reading rate) with which they are comfortable.

We have recently been using this moving window display while subjects also perform a crossmodal, dividedattention task. The subjects' secondary task is to respond as quickly as possible when they hear a tone. The Apple generates the tone through the Apple-Psych command

LISTING 3

Code to Create Simultaneous Visual and Auditory Stimuli

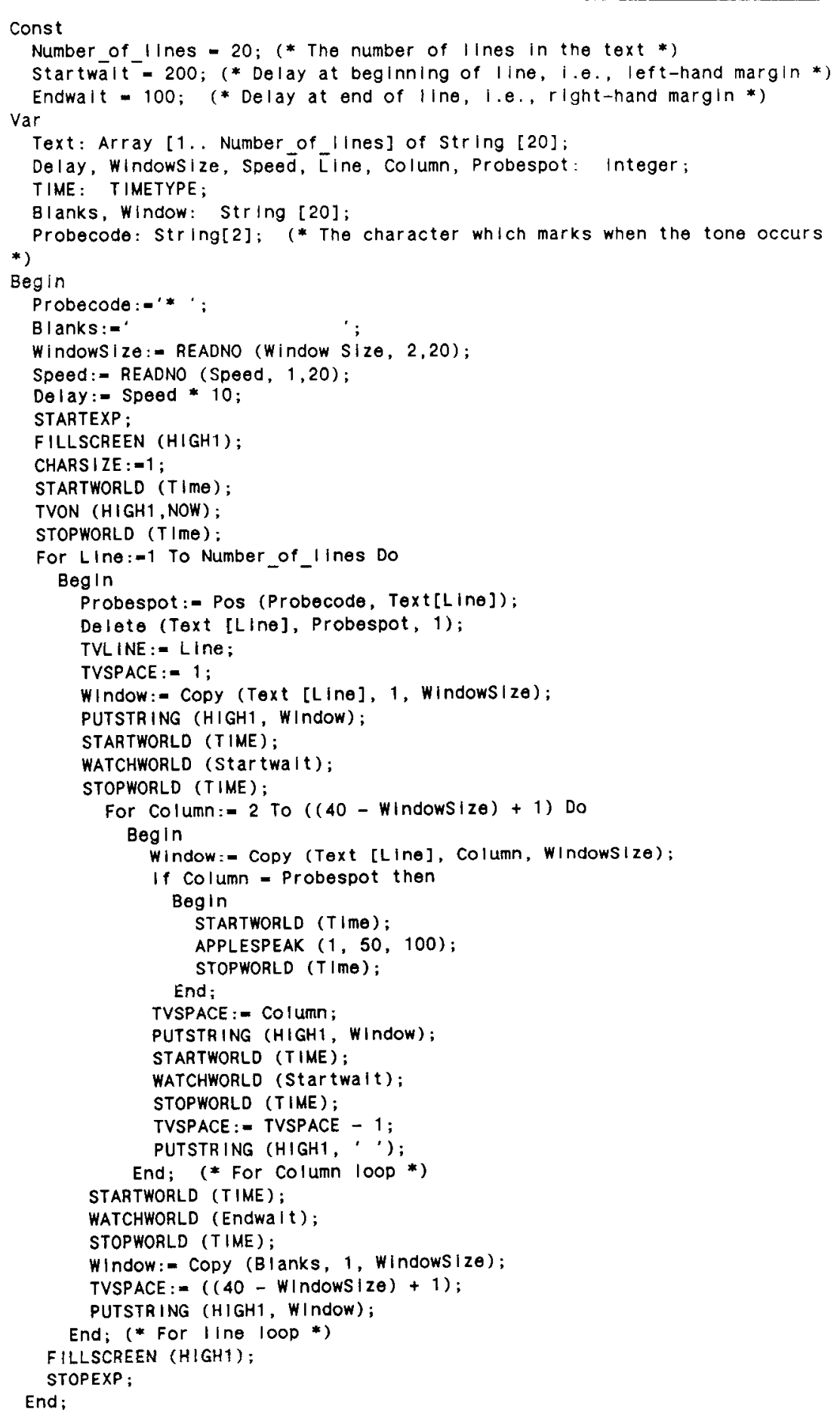


APPLESPEAK. In the stimulus file, we mark the place where we want the tone to occur with a prespecified character, for instance, an asterisk. Typically, we want the tone to occur coincident with the offset of the word or segment of text that we hypothesize to cause comprehension difficulty, for instance, after words with ambiguous meanings or pronouns that are difficult to find antecedents for. Listing 3 shows a portion of code to create this task.

A more traditional crossmodal task is Swinney's (1979) crossmodal lexical decision task. Subjects are auditorily presented with discourse and, at certain points during the auditory presentation, they perform a lexical decision on a visually presented string of letters (i.e., they decide rapidly whether the string of letters forms a word). In this case, the Apple via the Apple-Psych system is responsible for displaying the visually presented lexical decision stimuli and collecting the subjects' responses, but a prerecorded audiotape essentially "drives" the ApplePsych program. That is, the program waits for a signal from the audiotape before presenting the visual stimuli or recording the subjects' responses. To send such signals to the computer, we record separately on each of two audio channels. Text is recorded on Channel 1 . Then, while playing back Channel 1 , we simultaneously record high-frequency tones on Channel 2. The tones are produced by a square-wave generator, which outputs directly to Channel 2 of the tape.

The output from Channel 1, the channel containing the text, is presented binaurally to the subjects via their headphones. The output from Channel 2, the channel contain- ing the high-frequency tones, is sent through a voiceactivated relay that is connected to the parallel $\mathrm{I} / \mathrm{O}$ card of the Apple. This arrangement is illustrated in Figure 2. When the voice-activated relay senses a tone, it triggers the computer in the same manner as does a subject's keypress. The particular "keys" being triggered are designated in the program as distinct from subjects' responses. When the computer program reaches a point at which it is expecting a stimulus presentation, it essentially waits for the voice-activated relay to be triggered, and then it presents a stimulus item. Listing 4 shows a generic algorithm for such a presentation. Other tasks, such as verifications, can also occur in a crossmodal paradigm.

\section{Event-Related Potentials}

In collaboration with our colleagues at the University of Oregon, we are beginning to conduct language comprehension experiments in which the primary dependent variables are event-related potentials (ERPs). ${ }^{4}$ We have interfaced an Apple IIe with a Digital Equipment Corporation PDP 11-34. The Apple, using the Apple-Psych system, houses the master program that drives the experiment. The Apple is connected (via a RS232 serial interface) to the PDP-11, which collects 16 channels of EEG data following a command line in the Apple program. The Apple controls the stimulus presentation; however, because of memory limitations, the storage of the EEG data is the responsibility of the PDP-11. Gil Osgood has written the system routines for the PDP-11, which are called from the Apple program. These proce-

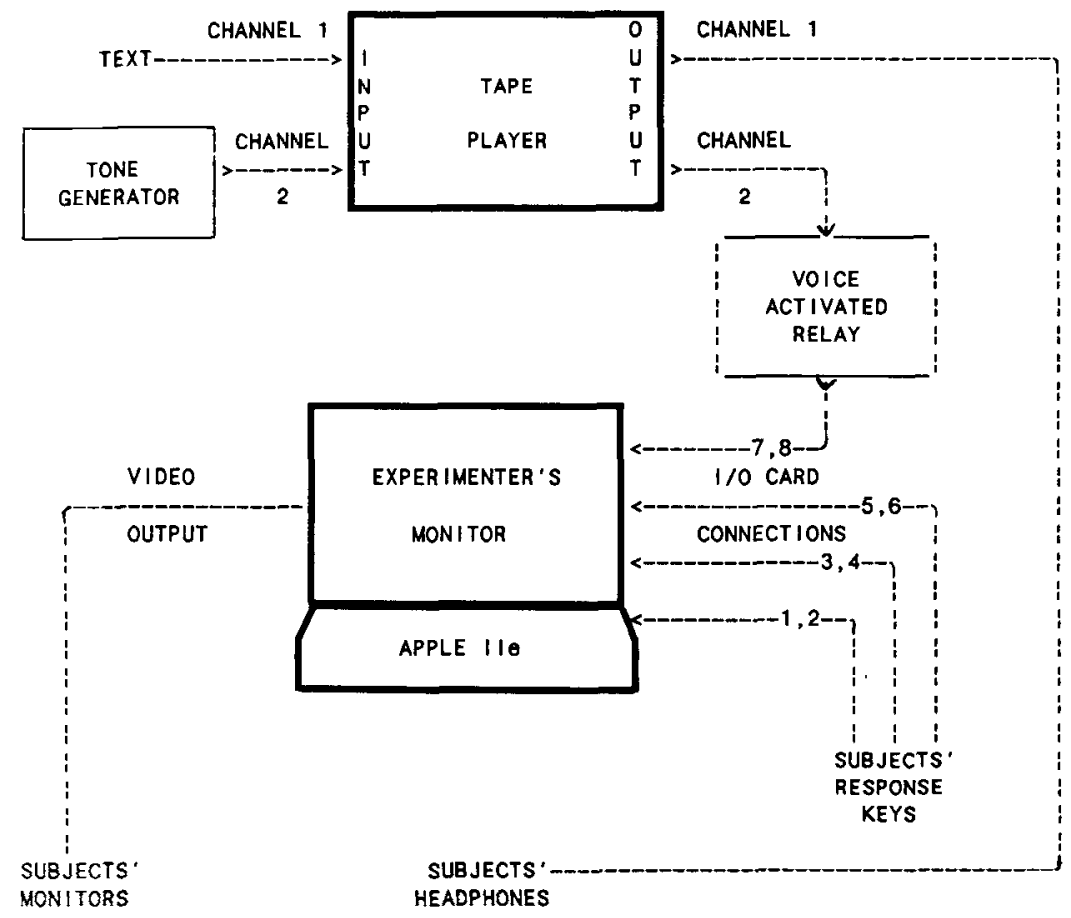

Figure 2. Schematic representation of the interfacing necessary to run 3 subjects on a crossmodal lexical decision task. 
LISTING 4

Code to Present Visual Stimuli in Response to Voice-Activated Relay

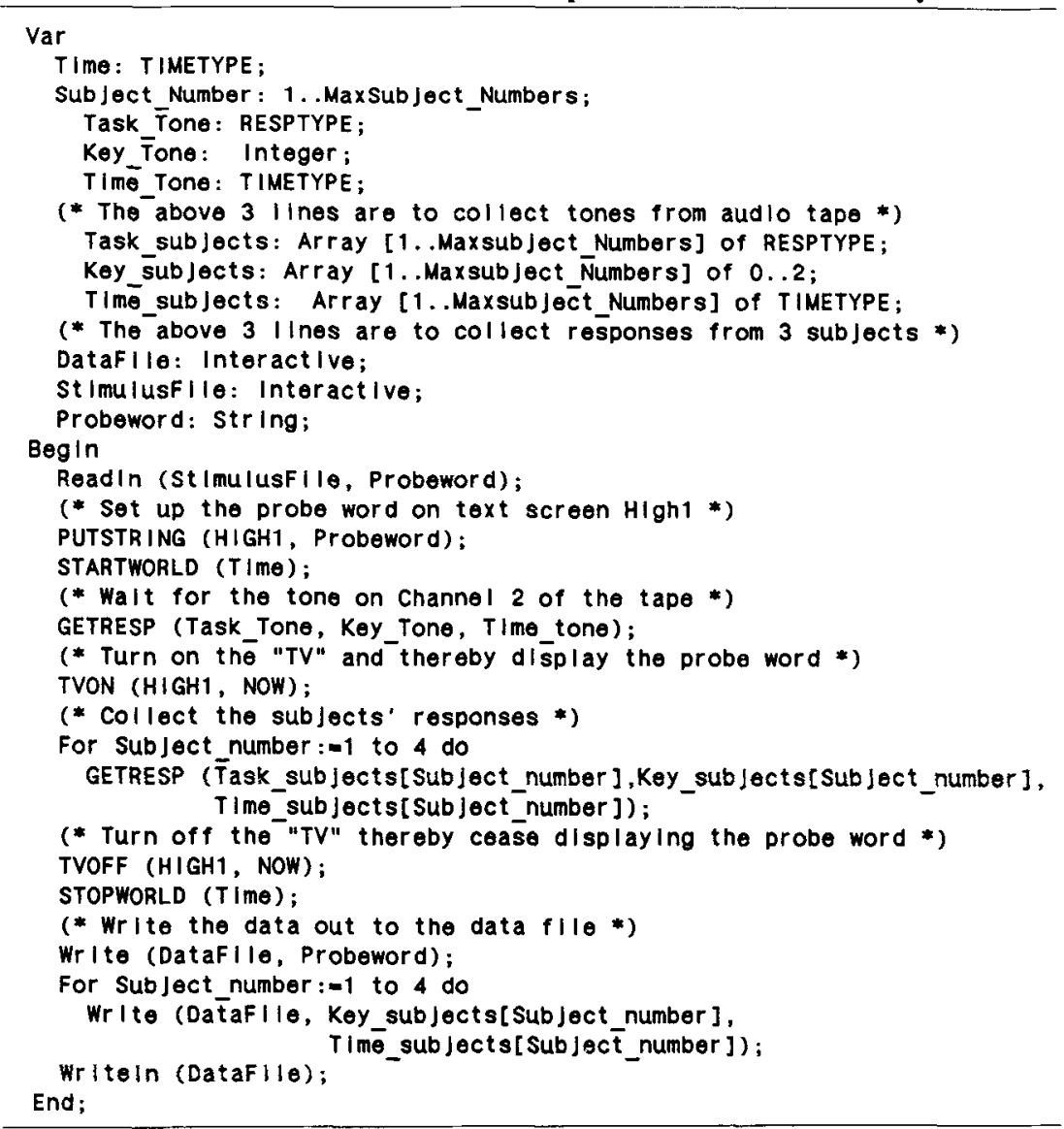

dures could be included in the Apple-Psych program if the computer had sufficient memory to store the EEG data. Such a setup will be possible when the Apple-Psych system is generalized for use on more powerful microcomputers (Osgood, 1988).

To run an ERP experiment, a data file on the PDP-11 must be created with the number of trials specified. Once the experiment begins, the communication link between the Apple and the PDP-11 is initiated. Once the link is established, the PDP-11 can collect EEG data, which are digitized waveforms, on command. For each second of EEG data collected, 256 samples are stored as digits ranging between $+1,024$ and $-1,024$. The command to begin collecting EEG samples is typically issued before the Apple presents the stimulus. Immediately after the command is issued, EEG collection begins, and control returns to the Apple so that other types of responses can be recorded or so that further stimuli can be presented. These commands consume very little memory and execution time; they, therefore, can be implemented on existing Apple-Psych programs.

Crossmodal paradigms are ideally suited for ERP experiments. Auditory presentation of text does not, of course, lead to the eye movements that create tremendous artifacts in EEG recordings. The Apple-Psych system allows us to drive the experiment by taped auditory dis- course, as described above. The same signal that causes presentation of a lexical decision or verification task can also trigger the collection of the EEG data, and thus the EEG data are time-locked to the stimulus discourse.

\section{Concluding Remarks}

The Apple-Psych system was created for running psychological experiments. It provides precision control over the timing of stimulus presentation and data collection. This permits fine-grained analyses of comprehension processes on-line, as they occur. In addition, the flexibility of the interfacing that is possible with the ApplePsych system allows for many useful modifications. Up to 4 subjects can be run simultaneously. Linguistic stimuli can be presented in many interesting ways, including visual and auditory presentation. Secondary tasks are easily tied to the discourse being comprehended, and additional types of data collection, such as event-related potentials, are possible.

\section{REFERENCES}

Aaronson, D., \& Scarborough, H. S. (1976). Performance theories for sentence coding: Some quantitative evidence. Journal of Experimental Psychology: Human Perception \& Performance, 2, 56-70. BARNes, S., \& BuRKe, R. S. (1988). What is Apple-Psych? Behavior Research Methods, Instruments, \& Computers, 20, 150-154. 
Chang, F. R. (1980). Active memory processes in visual sentence comprehension: Clause effects and pronominal reference. Memory \& Cognition, 8, 58-64.

Chang, F. R. (1983). Mental processes in reading: A methodological review. Reading Research Quarterly, 18, 216-230.

Juola, J. F., WARD, N., \& McNamara, T. (1982). Visual search and reading rapid serial presentations of letter strings, words, and text. Journal of Experimental Psychology: General, 111, 208-227.

Just, M. A., Carpenter, P. A., \& Woolley, J. D. (1982). Paradigms and processes in reading comprehension. Journal of Experimental Psychology: General, 111, 228-238.

KinTsCH, W., \& Mross, E. F. (1985). Context effects in word identification. Journal of Memory \& Language, 24, 336-349.

LEVELT, W. J. M. (1978). A survey of studies in sentence perception: 1970-1976. In W. J. M. Levelt \& G. B. Flores d'Arcais (Eds.), Studies in the perception of language. Amsterdam: North-Holland.

Oscood, G. (1984a). Hardware implementation and utility manual. Eugene, OR: University of Oregon.
OsGOOD, G. (1984b). Software program development guide. Eugene, $\mathrm{OR}$ : University of Oregon.

OsGood, G. (1988). Generalizing the Apple-Psych system. Behavior Research Methods, Instruments, \& Computers, 20, 155-157.

SWINNEY, D. (1979). Lexical access during sentence comprehension: (Re)consideration of context effects. Journal of Verbal Learning \& Verbal Behavior, 18, 645-659.

\section{NOTES}

1. Gil Osgood, Department of Psychology, University of Oregon, Eugene, OR 97403.

2. In each of these listings, the code written in capital letters is specific to the Apple-Psych system.

3. When measuring how long it takes subjects to read each successive piece of text, we typically test 1 subject at a time.

4. The primary researchers involved in this project are Don Tucker, Paul Compton, and Michael I. Posner. 